\title{
FOXP2 in focus: what can genes tell us about speech and language?
}

\author{
Gary F. Marcus ${ }^{1}$ and Simon E. Fisher ${ }^{2}$ \\ ${ }^{1}$ Department of Psychology, New York University, 6 Washington Place, New York, NY 10003, USA \\ ${ }^{2}$ Wellcome Trust Centre for Human Genetics, Oxford University, Roosevelt Drive, Headington OX3 7BN, UK
}

\begin{abstract}
The human capacity for acquiring speech and language must derive, at least in part, from the genome. In 2001, a study described the first case of a gene, FOXP2, which is thought to be implicated in our ability to acquire spoken language. In the present article, we discuss how this gene was discovered, what it might do, how it relates to other genes, and what it could tell us about the nature of speech and language development. We explain how FOXP2 could, without being specific to the brain or to our own species, still provide an invaluable entry-point into understanding the genetic cascades and neural pathways that contribute to our capacity for speech and language.
\end{abstract}

It has long been known that our genomes are, nucleotide for nucleotide, quite similar (around 98.5\%) to those of our nearest non-talking neighbors, chimpanzees [1,2]. Why is it that we can talk and they cannot? Culture must play some role, but chimpanzees that are raised in human environments do not acquire human linguistic competence, even with intensive tuition [3,4]. By contrast, groups of deaf children with restricted linguistic input can spontaneously create signing systems that have many hallmarks of natural spoken language, with structure at both word and sentence levels [5-7]. Over the years much evidence has accumulated to support the idea that aspects of our genetic makeup are critical for acquisition of spoken language [8]. Clearly some of the crucial changes are related to anatomical constraints [9]. For example, genetically encoded modifications of vocal tract morphology, which give us a longer oral cavity and lower larynx than other primates, are crucial for modern human speech, allowing us to produce a dramatically increased repertoire of sounds [9]. However, there are also likely to be genetic changes that impact on aspects of neurological development, including those that underlie our capacity for enhanced motor control over the articulators, as well as those that contribute to higher order cognitive processing involved in language acquisition and use [8].

\section{A gene mutated in a speech and language disorder}

A 2001 study identified the first case of a gene that may be implicated in our abilities for acquiring spoken language [10]. In humans, mutation of this gene, which has the technical name of 'FOXP2' (see Box 1) results in a severe

Corresponding authors: Gary F. Marcus (gary.marcus@nyu.edu) Simon E. Fisher (simon.fisher@well.ox.ac.uk). developmental disorder that significantly disrupts speech and language skills. The discovery of a link between FOXP2 and spoken language owes something to recent innovations in the field of molecular genetics [11], but was largely dependent on an opportune finding of a unique family known as KE [12]. This family consists of three generations in which about half of the members (15 individuals) suffer from severe speech and language difficulties (see Box 2 for discussion), although the remaining relatives escape unaffected. The inheritance of the problems in this family appears to be very straightforward, consistent with the involvement of just

\section{Box 1. FOXP2 hunting}

Even when family and twin-based data strongly suggest that genes play a role in a particular cognitive or behavioural trait, finding the particular gene or genes involved is a challenging task $[11,36]$. In the unusual case of the KE family, the inheritance of impairment follows the simple pattern of a single-gene disorder, rather than the more complex (and more difficult to trace) pattern of a trait that is caused by the interaction of multiple genes [11]. This suggested that the problems of the family were the result of damage to just one gene. In their search for the gene that was damaged, geneticists tried to correlate the distribution of a set of 'markers' - small stretches of DNA of known location that tend to vary between individuals - with the distribution of the disorder among members of the KE family [13]. This led them to a small region (or 'locus') of the long arm of chromosome 7. Although the markers themselves were not thought to be causally responsible for the disorder, they appeared to be in close proximity to the damaged gene and thus provided an index to its approximate chromosomal location (see Fig. 1 in main text).

The next step was to search that locus - more than 70 genes long to identify which of the genes that lay within it was damaged [14]. A chance discovery of another individual, who was not related to the KE family, but had a disorder with similar symptoms, greatly accelerated the process. This individual had a major chromosomal rearrangement, in which part of his chromosome 7 had broken and become attached to part of chromosome 5. The point of breakage on chromosome 7 lay in the middle of the critical region implicated in the KE family. More precise analysis showed that this breakpoint directly interrupted a gene that appeared to belong to a group of genes encoding forkhead transcription factors (see Box 3 ). The gene was given the name ' $F O X P 2$ ', which stands for Forkhead bOX P2, on the basis of comparisons with other forkhead genes. (There are multiple branches of the forkhead gene family, which are designated with letters from A-O. FOXP2 was the second gene to be identified in the ' $P$ ' branch.) Analysis of FOXP2 in the affected KE individuals revealed that they had all inherited a single nucleotide change in the gene sequence, which was likely to disrupt the function of the resulting protein [10]-establishing for the first time an unambiguous link between the mutation of a gene and a developmental disorder of speech and language. 


\section{Box 2. Debating deficits: motor control, language ability and the KE family}

Although the disorder that afflicts members of the KE family was first described in the scientific literature over a decade ago [12], the precise nature of the primary neurobiological deficit remains open to question. Hurst et al. first characterised the disorder as 'developmental verbal dyspraxia', in reference to the profound articulation difficulties experienced by affected individuals in early childhood [12]. However, Myrna Gopnik put forward a contrasting viewpoint proposing that the language problems of the KE family stem from an underlying deficit in specific grammatical abilities, such as the use of features marking number, gender and tense $[15,16]$. This 'featureblindness' hypothesis received widespread media attention and has sometimes been taken as evidence for existence of genes specific to grammar. However, extensive work by Faraneh Vargha-Khadem and colleagues has made it clear that the impairment in the KE family is not restricted to selective aspects of grammar [17-21]. In line with the original description of the KE family [12], Vargha-Khadem's team demonstrated that affected individuals have severe difficulties with controlling complex co-ordinated face and mouth movements (orofacial dyspraxia), which impedes their speech [18-20]. Furthermore, although the disorder does involve deficits in generating word inflections and derivations, impairment is broader than that suggested by Gopnik, affecting many aspects of grammar and language ability [18,21]. For example, affected individuals have significant problems in understanding sentences with complex syntactical structure (assessed by picture selection), in distinguishing real words from non-words (lexical decision), in reading and spelling non-words, and in manipulating phonemes [18,21].

Orofacial dyspraxia is a prominent aspect of the disorder, and one that could be considered a 'core' feature, in the sense that it gives a clear distinction between affected and unaffected members of the KE family. As such, some scholars have wondered whether the observed linguistic difficulties might be inevitable secondary consequences of a fundamental deficit in motor control, with no genuine relevance for higher cognition. There are several considerations that would suggest otherwise. First, the affected KE individuals perform normally for single simple oral movements and do not show abnormalities in limb praxis [19-21]. Second, it is true that the affected members have similar problems not just with speech but also with complex non-speech mouth movements; however the two are not significantly correlated with each other in these individuals, so the speech difficulties are not fully accounted for by more basic impairments in orofacial praxis [19]. Third, the disorder is not confined to production of spoken language; impairment is evident on tests of written language (such as those assessing verbal fluency) and, as outlined above, the affected members have trouble with language comprehension as well as production [18,21]. Fourth, recent brain imaging of affected $\mathrm{KE}$ members indicates functional abnormalities in language-related cortical regions, demonstrating that their problems extend beyond the motor system [34]. Clearly, the links between development of brain systems underlying speech motor control and those contributing to language abilities are highly complex and not yet well understood. A more complete explanation of the relation between the motor and linguistic aspects of the disorder might come from future integration of data from genetic, linguistic and neurophysiological studies [11].

a single gene on an autosome (that is, a chromosome but not one of the sex chromosomes), acting in a dominant fashion [13]. In itself, such an observation of monogenic inheritance is nothing extraordinary; several thousand different human disorders are known to be caused by changes in single genes (see http://www.ncbi.nlm.nih.gov/ $\mathrm{Omim} /$ ). However, the $\mathrm{KE}$ family remains the only documented case of simple inheritance for a developmental speech and language disorder, and by studying the DNA of affected and unaffected KE individuals [13,14] it

\section{Box 3. FOX transcription factors: regulating complexity}

Forkhead proteins are a set of evolutionarily-related transcription factors that perform a wide variety of functions in cellular differentiation and proliferation, pattern formation and signal transduction [22]. Many are implicated in regulating the development of the growing embryo, and some also have distinct roles in adult tissues, for example in controlling metabolism.

The first member of the forkhead group of transcription factors to be discovered was a gene that is essential for proper formation of terminal structures in fruit-fly embryos [37], and the name 'forkhead' relates to the unusual spiked-head structures found in fly embryos with mutations in that original gene. The uniting feature of all FOX proteins is the forkhead box, a small string of 80-100 amino acids forming a motif that binds to DNA [22]. This DNA-binding domain is a crucial element that allows FOX proteins to regulate expression of target genes (although other varying parts of each protein are also important). In the KE family it is the DNA-binding domain of FOXP2 that is mutated in those individuals affected with speech and language disorder.

Mutations of the genes encoding forkhead proteins have been implicated in diverse developmental disorders [22], including glaucoma (FOXC1), thyroid agenesis (FOXE1), immune deficiency (FOXN1 and FOXP3), ovarian failure (FOXL2) and lymphedema (FOXC2). For some FOX proteins it appears that the amount of functional protein (dosage) has an important influence on particular aspects of development $[22,38]$. For example, it has been shown that alteration of FOXC1 dosage has dramatic effects on the development of the eye [38].

FOX genes are only found in animals and fungi, and it is intriguing to note that there is a correlation between the number of different FOX genes in a genome and the anatomical complexity of the organism. Based on current available sequence information the yeast genome contains just four distinct $F O X$ genes, whereas 15 are found in the nematode, 20 in the fruit-fly, and at least 40 in human beings. Given their importance in patterning of embryos, it has been suggested that the expansion of this group of genes might have been driven by increasing body-plan complexity [22]. Perhaps one or more FOX genes might have played similar roles in generating aspects of neural complexity.

was possible to track down the specific mutation, which ultimately turned out to be an alteration of sequence in the FOXP2 gene [10] (see Box 1; Fig 1).

The disorder in the KE family, and in an unrelated individual with a gross chromosomal rearrangement involving FOXP2, is confined to the central nervous system and greatly impacts on the development of speech and language abilities. As outlined in Box 2, it involves multiple associated deficits, including articulation difficulties, language problems, and grammatical impairment [12,15-21]. Examination of the cognitive profiles of members of the KE family indicates that this is not a generalised intellectual delay. Although some affected individuals have a moderate reduction in non-verbal IQ, others are in the normal range despite their speech and language problems, and there is substantial overlap between IQ scores of the affected and unaffected family members [18,21]. Moreover, the observed deficits in verbal cognition are more severe and wide-ranging than those found on tests of non-verbal ability. For the affected individuals, Watkins and colleagues reported significant deficits with every sub-test used to assess verbal IQ, but only one out of four different non-verbal sub-tests [21]. The relative importance of different features of this form of speech and language disorder has been debated for over a 


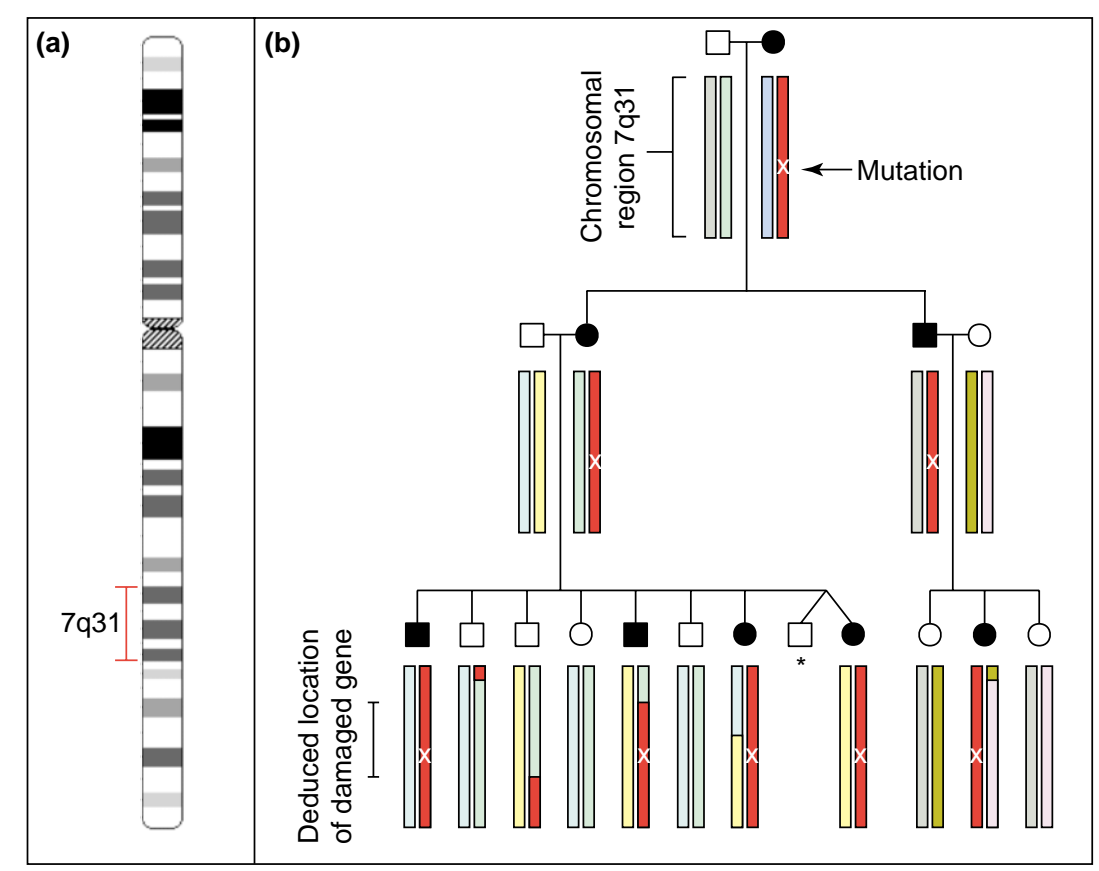

$\overline{\text { TRENDS in Cognitive Sciences }}$

Fig. 1. Locating the damaged gene in the KE family. For simplicity, only a subset of the family is shown (b), including two branches containing 8 of the total 15 affected individuals. (See Ref. [13] for a full pedigree diagram and more details.) Squares represent males, circles represent females, black shaded individuals are affected with speech and language disorder, unshaded individuals are normal. The star indicates a non-identical twin whose DNA was not available for study. Every human being inherits two copies of each chromosome (one paternal, one maternal), and sections of these copies get shuffled around from generation to generation by a process of exchange known as recombination. Thus, the genomic make-up that is inherited by an individual is best viewed as a patchwork of the maternal and paternal chromosomes from previous generations, and this can be exploited in order to locate damaged genes. Geneticists used information from a series of genetic markers to track the pattern of inheritance of chromosomal regions through the KE family [13]. They found particularly interesting results for one section of chromosome 7; part of the chromosomal band known as $7 q 31$ (a). In (b) different colours indicate the origins of different copies of chromosomes within the KE family. It was discovered that all affected individuals in the KE family had inherited an identical copy of one portion of 7q31. This copy originated from one chromosome of the affected grandmother (labelled in red). By determining the precise portion of the red-labelled copy of 7q31 that was common to all affected members (but not inherited by any unaffected members) it was possible to deduce the likely location of the damaged gene [13]. The researchers were then able to focus on genes from this specific portion of chromosome 7 in their subsequent mutation screening efforts [14] (and see Box 1), and eventually tracked down the mutation responsible [10] (denoted here by a white X).

decade, and, at the cognitive level, the question of a 'core deficit' remains controversial [12,15-21] (see Box 2). From the genetic point of view, however, there is no ambiguity; inheritance of a mutated copy of FOXP2 is necessary and sufficient to cause problems [10]. So, how does the knowledge of a clear genetic explanation help us to understand speech and language deficits at the neurological and cognitive levels? Can this discovery tell us anything in general about acquisition of spoken language?

\section{FOXP2 function-lessons from developmental biology}

Before we can properly address these and other related questions, we must gain some idea of the normal function of FOXP2. Fortunately, we can already make some educated guesses, by comparing the sequence of the gene to that of other genes that have been studied for several years and are thus better understood. On the basis of these comparisons, the FOXP2 gene belongs to a group of genes that make proteins containing forkhead-box (FOX) domains [22] (Box 3). FOX proteins are themselves just one subtype of a much larger group, known as transcription factors, which are involved in controlling the genetic programs of cells.

To understand the importance of transcription factors it is useful to view genes in terms of two key constituents; coding regions and regulatory regions (Fig. 2). The coding region of a gene comprises the template for the construction of a protein containing a specific sequence of amino acids. Protein-building relies on an initial step where the information contained in the DNA sequence of each gene is transcribed into intermediary copies (mRNA molecules), via a carefully controlled process called 'transcription'. The regulatory region of a gene is involved in determining how many copies of a gene's mRNA are made (and hence the amount of the encoded protein) in a particular cell at a given moment. Taken together, the two parts of a gene act something like an IF $\rightarrow$ THEN statement in a computer program; if certain conditions hold, then a protein is synthesized. Transcription factors, like that encoded by FOXP2, are proteins that interact with the regulatory regions of genes and modulate their transcription levels, thereby ultimately influencing the relative abundances of other proteins in the cell. Overall, the system enables the cells of an organism to dramatically diversify their morphology and function by modifying the levels of expression of different genes in a tissue-specific manner, at particular points during the organism's development, or in response to internal/external stimuli.

One of the most well-studied of transcription factor genes is one that is known as PAX6 [23,24]. PAX6 plays a crucial part in the development of the eye, and an understanding of its role might give some insight into 


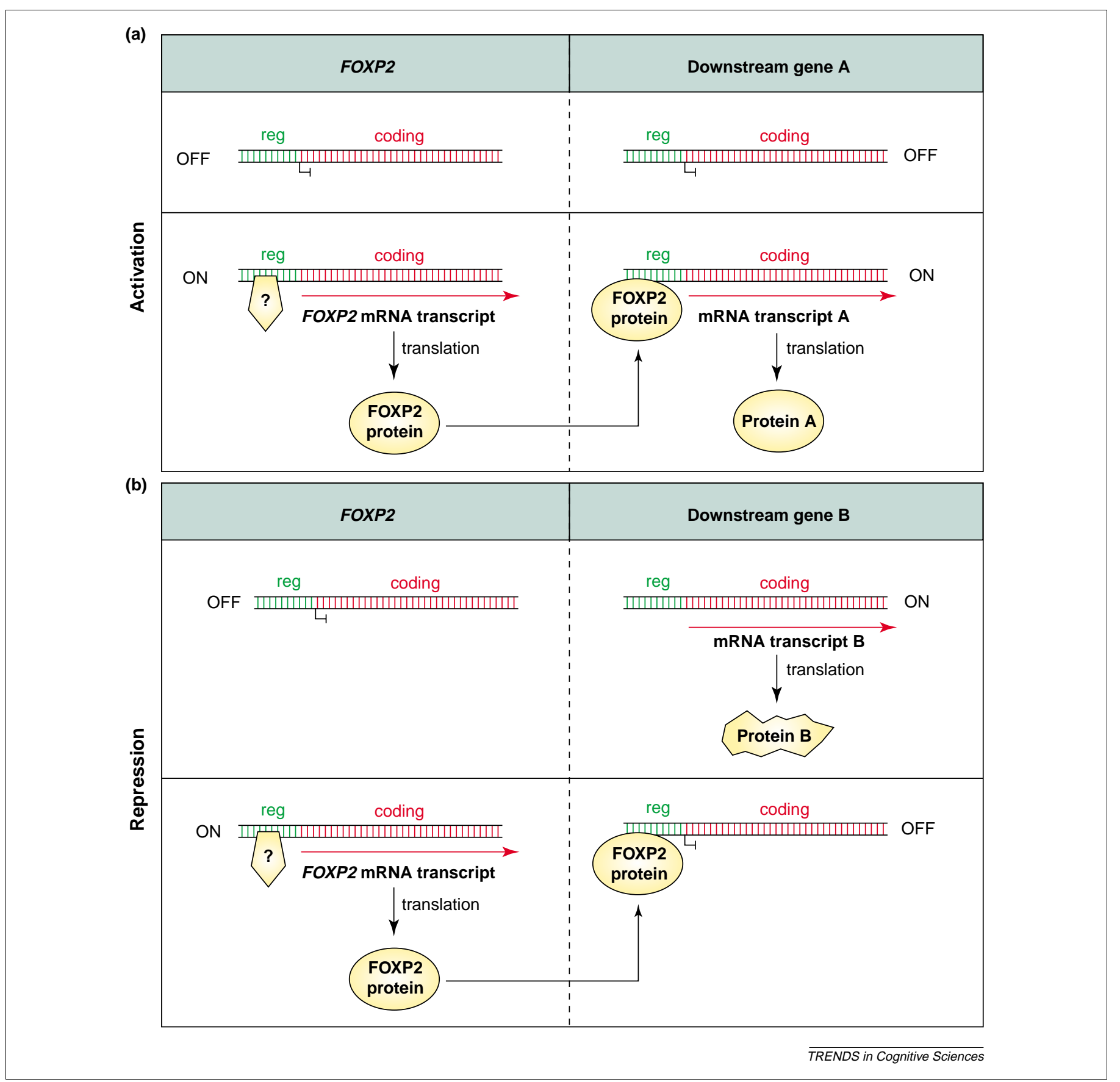

Fig. 2. What is the function of FOXP2? On the basis of currently available data, the FOXP2 protein probably acts as a transcription factor, binding to regulatory regions (green) in the genomic DNA of target genes, and modulating the rate at which the coding regions of those genes (red) are transcribed into messenger RNA (mRNA), which in turn serve as intermediate temPlate that are then translated into proteins (yellow). The figure depicts two possible ways that transcription factors can influence the expression of a target gene: (a) Activation (which increases the transcription of a target gene); hypothetical scenario illustrated in which FOXP2 stimulates transcription of target gene ' $\mathrm{A}$ '; and (b) Repression (which decreases the transcription of a target gene); scenario in which FOXP2 inhibits transcription of target gene 'B'. In reality, FOXP2 might influence the expression of many more genes, and its influence is very likely to be mediated by interactions with other proteins. (We have further simplified by ignoring additional complexities of transcription and translation, and by depicting genes here as simply being in alternate states of 'on' or 'off', whereas in fact transcriptional regulation can involve much more subtle changes in gene expression.) Future work will aim to determine the identities of the proteins (indicated by '?') that act upstream of FOXP2 to control its expression, the actual targets of FOXP2, and the co-factors that interact with the FOXP2 protein to regulate its function. Experiments have demonstrated that FOXP2 is able to repress certain lung-specific genes [26], but its role in activation/repression of genes involved in neurogenesis or neuronal function is still unknown [11].

possible functions of FOXP2. When PAX6 is artificially expressed in the antenna of a fruit-fly, the fly grows an extra eye right there, at that rather unusual location [25]; for this reason, Walter Gehring has described it as a 'master control gene' [23]. PAX6 by itself does not actually contain a blueprint or plan for eye-building. Instead, it promotes eye development by mobilizing - through direct and indirect action - a vast number of genes, perhaps 2500 in all [25]. Like a Chief Executive Officer, it might not do much work on its own, but its orders can determine the actions of many subordinates, referred to in biology as downstream targets. Even without knowing which subordinates receive orders from $P A X 6$, the gene's importance is obvious. Still, we cannot be said to fully appreciate either 
its function or the process of eye development as a whole until we understand not just PAX6 but all of the genes it interacts with, directly and indirectly, the organizational chart as a whole. The situation is likely to be similar with FOXP2, its power coming from the consequences it has for a cascade (or cascades) of other genes.

\section{Building brains... and lungs, guts and hearts}

FOXP2 is expressed in restricted parts of the developing brain and might participate in generating neural substrates involved in acquisition of spoken language. But FOXP2 is also expressed in defined regions of other tissues during embryo development, including the lung, the gut and the heart [26], as well as in several tissues of the adult organism [10]. Again, this has parallels in what we already know about other transcription factors, many of which have multiple differing jobs, sometimes at diverse timepoints during the lifetime of an organism. For example, our 'master control gene' for eye-building, PAX6, is also essential for development of the central nervous system and endocrine glands, and regulates a range of cellular processes, including proliferation, migration, adhesion and signalling [24].

Why are the deficits of the KE family confined to brain function, if the gene is so important for development of organs like the lung or heart? The answer to that question could lie in the fact that our genome is diploid-we each have two copies of every gene (one from our mother and one from our father). The problems of the $\mathrm{KE}$ family are associated with mutation in just one copy of the FOXP2 gene, which probably leaves affected members with only half the usual dosage of normally functioning FOXP2 protein. This dose might be adequate for development of the lungs, gut and heart, but insufficient for the brain (or certain sub-structures and/or cell-types within). Although we cannot be certain that the effects of altered dosage are indeed the explanation, there are again many precedents for its importance in studies of other transcription factors, including several other types of forkhead gene [22] (see Box 3). Alternatively, there might be subtle changes in tissues other than the brain that we have not yet been able to detect.

\section{Evolving FOXP2}

Just as FOXP2 is not unique to the brain, it is not unique to Homo sapiens. Mice, for example, have a version of the gene with a nucleotide coding sequence that is $93.5 \%$ identical to the human version [27], and yet (except in the world of cartoons and children's films) no mouse has ever been heard to speak. Does the observation that FOXP2 is present in a similar form in a broad range of mammalian species imply that it is not involved in speech and language after all? Not at all. If the rest of biology is any guide, the machinery of language is likely to be the product of a mixture of evolutionary novelty and evolutionary recycling. In general, the way that new structures are built is by small (but sometimes significant) modifications of old machinery. As Francois Jacob put it, evolution is like a tinkerer who 'often without knowing what he is going to produce, ... uses what ever he finds around him, old cardboards, pieces of strings, fragments of wood or metal, to make some kind of workable object...' [28]. The genetic mechanisms involved in speech and language development are likely to involve recruitment and modification of pre-existing genetic cascades, much in the way that the development of the wing began with the development of the basic design of a vertebrate forelimb [29]. Speech and language, in this sense, may derive in part from unique configurations of genetic cascades that figure in other neural systems, such as those that direct motor control, planning, social cognition, and spatiotemporal representation.

Investigations of mouse FOXP2 [26] suggest that the gene was already playing a significant role in the development of the brain in the common ancestor of mice and humans (perhaps, for example, in patterning of neural structures involved in aspects of motor control). Only three amino acid differences distinguish the versions of FOXP2 protein found in mouse and man, but two of these changes occurred on the human lineage after separation from the human-chimp common ancestor $[27,30]$. Computer-based comparisons of human and chimp protein sequences indicate that one of these changes may have had important consequences for FOXP2 function, by altering the way it is regulated by other proteins. Intriguingly, mathematical analyses of the within-species variability of the FOXP2 genomic locus suggest that FOXP2 has been a target of selection relatively recently in human history $[27,30]$. These studies concluded that modern human-specific FOXP2 most likely became fixed in the human population within the last 200000 years, consistent with several archaeological estimates of the time of emergence of proficient spoken language [31,32].

Overall, the presence of FOXP2 in other animals does not diminish its relevance for speech and language, but rather represents another example of recruitment and modification of existing pathways in evolution. Although the genetic pathways implicated in language may have in part been recruited from genetic cascades involved in other brain systems, this does not mean that they necessarily draw on the same neural substrates as these other systems. It is quite possible, for example, that the development of an ability to represent hierarchical grammar might be influenced by some of the same genes as those influencing representation of hierarchical plans in other domains, even if the circuitry involved were physically located in two places. Separate circuits in separate brain areas could compute similar functions, allowing, for example, the simultaneous planning of a linguistic sentence and a motor sequence. Coming back to FOXP2 and the KE family, it might be the case (as discussed in Box 1) that a deficit restricted to the motor system is fully responsible for the wide-ranging profile of impairment. However, it is also possible that the different aspects of the disorder are separate consequences of disruption to a gene that is expressed in separate systems. Consistent with this idea, neuroimaging studies have detected structural and functional anomalies in several different brain regions in affected members of the $\mathrm{KE}$ family [19,33,34]. These abnormalities include a bilateral reduction of grey matter density in the caudate nucleus (a site of pathology that could account for the motor aspects of 
the disorder) [19,33], as well as significant underactivation in several language-related regions, including Broca's area, during covert and overt word generation tasks [34].

\section{Uncovering neural pathways}

FOXP2 cannot be called 'the gene for speech' or 'the gene for language'. It is just one element of a complex pathway involving multiple genes, and it is too early to tell whether its role within that pathway is special. Furthermore, FOXP2 appears to be normal in common forms of developmental language disorder, and these seldom involve the kinds of oromotor deficits observed in the $\mathrm{KE}$ family [35]. Nonetheless, the gene can provide a valuable entry-point into the relevant neural pathway (or pathways), by pointing to the downstream targets which it regulates or the proteins with which it interacts. Speech and language are likely to be products of mechanisms that are shared with other neural domains and those that are not. They are also likely to represent a mixture of genetic mechanisms, including those that are found to vary among normal individuals and those that are not. A gene like FOXP2 that is virtually invariant in the normal population $[27,30,35]$, could cast light on both sorts of mechanisms: directly, on invariant processes in which it participates, and indirectly, by allowing the identification of other elements of these pathways that might vary between individuals. In each way, future studies of FOXP2 could provide a significant wedge into the understanding of our unique linguistic heritage.

\section{Acknowledgements}

G.F.M. was supported by the NIH, the HFSP, the MacArthur Foundation and the Center for Advanced Study in Behavioral Sciences. S.E.F. is a Royal Society Research Fellow.

\section{References}

1 King, M.C. and Wilson, A.C. (1975) Evolution at two levels in humans and chimpanzees. Science 188, 107-116

2 Ebersberger, I. (2002) Genomewide comparison of DNA sequences between humans and chimpanzees. Am. J. Hum. Genet. 70, $1490-1497$

3 Hayes, K.J. and Nissen, C.H. (1971) Higher mental functions of a home-raised chimpanzee. In Behaviour of Non-human Primates (Schrier, A.M. and Stollnitz, F., eds) pp. 59-115, Academic Press, New York

4 Terrace, H.S. et al. (1979) Can an ape create a sentence? Science 206 , 891-902

5 Goldin-Meadow, S. and Feldman, H. (1977) The development of language-like communication without a language model. Science 197, 401-403

6 Goldin-Meadow, S. and Mylander, C. (1998) Spontaneous sign systems created by deaf children in two cultures. Nature 391, 279-281

7 Senghas, A. and Coppola, M. (2001) Children creating language: how Nicaraguan sign language acquired a spatial grammar. Psychol Sci. $12,323-328$

8 Pinker, S. (1994) The Language Instinct, Allen Lane

9 Fitch, W.T. (2000) The evolution of speech: a comparative review. Trends Cogn. Sci. 4, 258-267

10 Lai, C.S.L. et al. (2001) A novel forkhead-domain gene is mutated in a severe speech and language disorder. Nature 413, 519-523
11 Fisher, S.E. et al. (2003) Deciphering the genetic basis of speech and language disorders. Annu. Rev. Neurosci. 26, 57-80

12 Hurst, J.A. et al. (1990) An extended family with a dominantly inherited speech disorder. Dev. Med. Child Neurol 32, 352-355

13 Fisher, S.E. et al. (1998) Localisation of a gene implicated in a severe speech and language disorder. Nat. Genet. 18, 168-170

14 Lai, C.S.L. et al. (2000) The SPCH1 region on human 7q31: genomic characterization of the critical interval and localization of translocations associated with speech and language disorder. Am. J. Hum. Genet. 67, 357-368

15 Gopnik, M. (1990) Feature-blind grammar and dysphasia. Nature 344 , 715

16 Gopnik, M. and Crago, M.B. (1991) Familial aggregation of a developmental language disorder. Cognition 39, 1-50

17 Vargha-Khadem, F. and Passingham, R.E. (1990) Speech and language defects. Nature 346, 226

18 Vargha-Khadem, F. et al. (1995) Praxic and nonverbal cognitive deficits in a large family with a genetically transmitted speech and language disorder. Proc. Natl. Acad. Sci. U. S. A. 92, 930-933

19 Vargha-Khadem, F. et al. (1998) Neural basis of an inherited speech and language disorder. Proc. Natl. Acad. Sci. U. S. A. 95, 12695-12700

20 Alcock, K.J. et al. (2000) Oral dyspraxia in inherited speech and language impairment and acquired dysphasia. Brain Lang. 75, 17-33

21 Watkins, K.E. et al. (2002) Behavioural analysis of an inherited speech and language disorder: comparison with acquired aphasia. Brain 125, 452-464

22 Carlsson, P. and Mahlapuu, M. (2002) Forkhead transcription factors: key players in development and metabolism. Dev. Biol. 250, 1-23

23 Gehring, W.J. and Ikeo, K. (1999) Pax 6: mastering eye morphogenesis and eye evolution. Trends Genet. 15, 371-377

24 Simpson, T.I. and Price, D.J. (2002) Pax6; a pleiotropic player in development. Bioessays 24, 1041-1051

25 Halder, G. et al. (1995) Induction of ectopic eyes by target expression of the eyeless gene in Drosophila. Science 267, 1788-1792

$26 \mathrm{Shu}$, W. et al. (2001) Characterization of a new subfamily of wingedhelix/forkhead (Fox) genes that are expressed in the lung and act as transcriptional repressors. J. Biol. Chem. 276, 27488-27497

27 Enard, W. et al. (2002) Molecular evolution of FOXP2, a gene involved in speech and language. Nature $418,869-872$

28 Jacob, F. (1977) Evolution and tinkering. Science 196, 1161-1166

29 Gerhart, J. and Kirschner, M. (1997) Cells, Embryos, and Evolution, Blackwell Science

30 Zhang, J. et al. (2002) Accelerated protein evolution and origins of human-specific Features. Foxp2 as an example. Genetics 162, $1825-1835$

31 Boyd, R. and Silk, J.B. (2000) How Humans Evolved, W.W. Norton, New York

32 Klein, R.G. and Edgar, B. (2002) The Dawn of Human Culture, Wiley, New York

33 Watkins, K.E. et al. (2002) MRI analysis of an inherited speech and language disorder: structural brain abnormalities. Brain 125, $465-478$

34 Liegois, F.J. et al. (2002) Functional abnormalities associated with the FOXP2 (SPCH1) mutation in the KE family: an overt speech FMRI study. Soc. Neurosci. Abstr. Program No. 17.12. (Abstract Viewer, online)

35 Newbury, D.F. et al. (2002) FOXP2 is not a major susceptibility gene for autism or specific language impairment. Am. J. Hum. Genet. 70, $1318-1327$

36 Fisher, S.E. and DeFries, J.C. (2002) Developmental dyslexia: genetic dissection of a complex cognitive trait. Nat. Rev. Neurosci. 3, 767-780

37 Weigel, D. et al. (1989) The homeotic gene fork head encodes a nuclear protein and is expressed in the terminal regions of the Drosophila embryo. Cell 57, 645-658

38 Nishimura, D.Y. et al. (2001) A spectrum of FOXC1 mutations suggests gene dosage as a mechanism for developmental defects of the anterior chamber of the eye. Am. J. Hum. Genet. 68, 364-372 